\title{
Transdifferentiation of differentiated stem cells contributes to remyelination
}

\author{
Bharath Chelluboina', Dzung H. Dinh ${ }^{2,3}$ and Krishna Kumar Veeravalli ${ }^{*}$ \\ See related research by Qiu et al., http://www.stemcellres.com/content/6/1/105
}

\begin{abstract}
Evidence suggests that transdifferentiation of mesenchymal stem cells (MSCs) into various neuronal cells contributes to functional recovery after experimental spinal cord injury. Qiu et al. have recently published an exciting article in Stem Cell Research \& Therapy demonstrating the transdifferentiation of already differentiated MSCs that contributes to remyelination of injured/regenerating axons, and thereby to functional recovery of spinal cord injured animals. The authors highlight the importance of interaction between neurotrophin-3 and tropomyosin receptor kinase $\mathrm{C}$ for the observed effects. This study provided important evidence that manipulation of rat bone marrow-derived MSCs before transplantation could enhance the therapeutic benefit of cell-based treatment.
\end{abstract}

\section{Introduction}

Despite exciting progress in the field of regeneration, functional remyelination has not been demonstrated in the adult injured central nervous system. For transplanted mesenchymal stem cells (MSCs) to effectively reverse traumatic spinal cord injury (SCI), multiple barriers have to be overcome such as low survival of transplanted MSCs, limited neuronal transdifferentiation, remyelination, synaptogenesis, and, not least of all, gliosis. Regardless of the source of MSCs (adipose, bone marrow, or umbilical cord blood), cell-based therapy appeared to be promising in mitigating the extent of SCI, promoting neuronal repair and regeneration. Even though our laboratory has demonstrated the ability of MSCs to transdifferentiate and remyelinate injured axons, the

\footnotetext{
* Correspondence: krishnav@uic.edu

${ }^{1}$ Department of Cancer Biology and Pharmacology, University of Illinois

College of Medicine at Peoria, 1 Illini Drive, Peoria, IL 61605, USA

Full list of author information is available at the end of the article
}

mechanism behind these beneficial effects remains unclear [1-3].

\section{Main text}

Several recent studies have shown that manipulated Schwann cells (SCs) and MSCs which overexpressed neurotrophin-3 (NT-3) and tropomyosin receptor kinase $\mathrm{C}(\operatorname{TrkC})$, alone or in concert in co-cultured milieu, promoted survivability, neuronal transdifferentiation, remyelination, and synaptogenesis and attenuated the gliosis process [4-8]. This study by Qiu et al. [9] used only MSCs, instead of mixed SCs and MSCs as used in other studies. The rat mesenchymal stem cells (rMSCs) were genetically manipulated to overexpress either NT-3 or its high-affinity receptor TrkC and allowed the cells to differentiate in a three-dimensional gelatin sponge scaffold. Fourteen days after culture in the scaffold, none of the rMSCs was positive for astrocyte and oligodendrocyte markers. More than $70 \%$ of either manipulated or nonmanipulated rMSCs expressed immature neuronal markers. However, only $7 \%$ of the nonmanipulated rMSCs transdifferentiated into mature neuronal marker, as compared with $44 \%$ and $45 \%$ in NT-3-overexpressed rMSCs and TrkC-overexpressed rMSCs, respectively. The percentage of rMSCs positive for mature neuronal marker increased to $68 \%$ ( $p<0.05$ vs. other groups) when NT-3-overexpressing and TrkC-overexpressing rMSCs were cultured together. This study highlighted the importance of NT-3/TrkC interaction in improving the transdifferentiating potential of rMSCs to neural-like cells. Although these results are interesting, we still do not understand why the nonmanipulated rMSCs were positive only for immature neuronal marker and not for astrocyte or oligodendrocyte markers.

NT-3 is a member of one of the four known neurotrophins, which include nerve growth factor, brain-derived neurotrophic factor, and neurotrophin 4/5. NT-3 acts very strongly on neurons within the corticospinal tract [10]. Binding of NT-3 to its high-affinity receptor TrkC 
has been shown to trigger axonal growth, maturation, and plasticity of synapses [11]. Binding of NT-3 to TrkC also induces phosphorylation of intracellular tyrosine residues that block caspase activation associated with cell death [12]. Qiu et al. have provided evidence that NT-3/TrkC binding caused the transdifferentiation of already differentiated rMSCs in the injured rat spinal cord. The most interesting outcome of this study is the transdifferentiation of rMSC-derived neural-like cells into myelin-forming cells after their transplantation in the injured rat spinal cord. Here, the authors highlighted the importance and contribution of NT-3/TrkC interaction to the loss of neural phenotypes in rMSC-derived neural-like cells, the transdifferentiation of the differentiated rMSCs into myelin-forming cells, the formation of myelin sheaths around the injured/regenerating axons, and the locomotor recovery of spinal cord injured rats. However, the authors did not elucidate the underlying mechanism of transdifferentiation of already differentiated cells. It could be speculated that the local microenvironment of the demyelinated region may be more conducive to induce the transdifferentiation of rMSCderived neural-like cells into oligodendrocytes. Another study published by the same group of researchers demonstrated that the addition of exogenous NT-3 into the cultured TrkC-overexpressing rMSCs promoted their transdifferentiation into oligodendrocytes [7]. Currently, we do not know why NT-3/TrkC binding in vitro led to transdifferentiation of TrkCoverexpressing rMSCs into myelin-forming cells and the associated mechanisms. It is possible that binding of NT-3/TrkC activates downstream signaling of the mitogen-activated protein kinase kinase (MEK) -extracellular signal-regulated kinase (ERK) pathway [13].

\section{Conclusions}

Further studies are warranted to better understand the underlying molecular mechanisms of NT-3/TrkC signaling that leads to transdifferentiation of MSCs.

\footnotetext{
Abbreviations

MSC: Mesenchymal stem cell; NT-3: Neurotrophin-3; rMSC: Rat mesenchymal stem cell; SC: Schwann cell; SCl: Spinal cord injury; TrkC: Tropomyosin receptor kinase $C$.
}

\section{Competing interests}

The authors declare that they have no competing interests.

Acknowledgements

The authors thank Christina Constantinidou for manuscript preparation and review.

\section{Author details}

${ }^{1}$ Department of Cancer Biology and Pharmacology, University of Illinois College of Medicine at Peoria, 1 Illini Drive, Peoria, IL 61605, USA. ${ }^{2}$ Department of Neurosurgery, University of Illinois College of Medicine at Peoria, 530 N.E. Glen Oak Ave., Peoria, IL 61637, USA. ${ }^{3}$ Illinois Neurological Institute, OSF Saint Francis Medical Center, 530 N.E. Glen Oak Ave., Peoria, IL 61637, USA.
Published online: 05 October 2015

\section{References}

1. Dasari VR, Spomar DG, Gondi CS, Sloffer CA, Saving KL, Gujrati M, et al. Axonal remyelination by cord blood stem cells after spinal cord injury. J Neurotrauma. 2007;24:391-410.

2. Dasari VR, Spomar DG, Cady C, Gujrati M, Rao JS, Dinh DH. Mesenchymal stem cells from rat bone marrow downregulate caspase-3-mediated apoptotic pathway after spinal cord injury in rats. Neurochem Res. 2007;32:2080-93.

3. Veeravalli KK, Dasari VR, Fassett D, Dinh DH, Rao JS. Human umbilical cord blood-derived mesenchymal stem cells upregulate myelin basic protein in shiverer mice. Stem Cells Dev. 2011;20:881-91.

4. Wang JM, Zeng YS, Wu JL, Li Y, Teng YD. Cograft of neural stem cells and schwann cells overexpressing TrkC and neurotrophin-3 respectively after rat spinal cord transection. Biomaterials. 2011;32:7454-68.

5. Zhang $Y Q$, He LM, Xing $B$, Zeng $X$, Zeng CG, Zhang W, et al. Neurotrophin-3 gene-modified Schwann cells promote TrkC gene-modified mesenchymal stem cells to differentiate into neuron-like cells in poly(lactic-acid-co-glycolic acid) multiple-channel conduit. Cells Tissues Organs. 2012;195:313-22.

6. Lai BQ, Wang JM, Ling EA, Wu JL, Zeng YS. Graft of a tissue-engineered neural scaffold serves as a promising strategy to restore myelination after rat spinal cord transection. Stem Cells Dev. 2014;23:910-21.

7. Ding Y, Zhang RY, He B, Liu Z, Zhang Z, Ruan JW, et al. Combination of electroacupuncture and grafted mesenchymal stem cells overexpressing TrkC improves remyelination and function in demyelinated spinal cord of rats. Sci Rep. 2015;5:9133.

8. Zeng X, Qiu XC, Ma YH, Duan JJ, Chen YF, Gu HY, et al. Integration of donor mesenchymal stem cell-derived neuron-like cells into host neural network after rat spinal cord transection. Biomaterials. 2015;53:184-201.

9. Qiu XC, Jin H, Zhang RY, Ding Y, Zeng X, Lai BQ, et al. Donor mesenchymal stem cell-derived neural-like cells transdifferentiate into myelin-forming cells and promote axon regeneration in rat spinal cord transection. Stem Cell Res Ther. 2015;6:105.

10. Escandon E, Soppet D, Rosenthal A, Mendoza-Ramirez JL, Szonyi E, Burton $L E$, et al. Regulation of neurotrophin receptor expression during embryonic and postnatal development. J Neurosci. 1994;14:2054-68.

11. Poo MM. Neurotrophins as synaptic modulators. Nat Rev Neurosci. 2001;2:24-32.

12. Lodish H, Berk A, Zipursky S, Matsudaira P, Baltimore D, Darnell J. Molecular cell biology. 4th ed. New York: W. H. Freeman; 2000. p. 929.

13. Wang TT, Tio M, Lee W, Beerheide W, Udolph G. Neural differentiation of mesenchymal-like stem cells from cord blood is mediated by PKA. Biochem Biophys Res Commun. 2007;357:1021-7. 